\title{
Differential universes in external dynamic linearization
}

\author{
Zbigniew Bartosiewicz \\ bartos@cksr.ac.bialystok.pl \\ Ewa Pawłuszewicz \\ epaw@cksr.ac.bialystok.pl
Institute of Mathematics \& Physics, Białystok Technical University
Wiejska 45a, 15-351 Białystok, Poland
tel. +48857428200

\begin{abstract}
It is shown that a nonlinear continuous-time control system with output is externally dynamically linearizable if and only if its differential output universe is free. Some regularity conditions are assumed, but the system does not have to be observable. Using universes instead of algebras or fields is essential as they allow for substitutions and amalgamations of functions.

Keywords: external dynamic linearization, differential output universe, nonlinear control system with output.
\end{abstract}

Regular paper

\section{Introduction}

Dynamic state feedback linearization has been studied for several years by many authors. To obtain a linear system one uses a dynamical system in a feedback loop (e.g. [3]) or state and control transformations that involve derivatives of states and outputs (e.g. [4, 9]). We consider here systems with output and are interested in linearizing the external behavior of the system using dynamic transformation of output and control. The concept of flatness [6] is close to our investigations as flat output is close to free generator of a universe - the main concept we use here (see $[1,5])$. The difference lies in the problem: we are interested in transformation of output and control and not state and control. This requires getting state from output and control. The tools available in differential algebra are not enough to achieve this in an analytic case (see Example 6.4). Besides algebraic operations we need also substitutions into analytic functions and amalgamation (glueing-up) of partially defined functions. These are exactly the operations that constitute function universe.

We show that the differential output universe of a system is the only invariant of external dynamic equivalence. This is an extension of the result of Jakubczyk 
[4] which says that the differential algebra of a system without output is the only invariant of dynamic (state) feedback equivalence. In this case one can put $y=x$ (the output is the entire state) and the universe may be replaced by the algebra. On the other hand our characterization of external dynamic equivalence is parallel to our earlier result for discrete-time system $[2,7]$ where the invariant was the difference output universe. The idea of the proof for the continuous-time case is similar to that of the discrete-time case so we omit the proof.

We do not have to assume observability as we show that the quotient system with respect to the indistinguishability relation has the same differential output universe as the original (unobservable) system and the two systems are externally dynamically equivalent. Thus the main construction can only be done for an observable system which allows for recovering the state from the output and the control.

The main result of this paper is an application of the theorem on equivalence and it states that a nonlinear continuous-time system with output is externally dynamically linearizable if and only if its differential output universe is free. This is again parallel to the discrete-time version given in [8]. A simple example at the end shows that to find free generators of the system we need to perform substitutions and amalgamations.

\section{External dynamic equivalence}

Let us consider an analytic continuous-time control system defined on $\mathbb{R}^{n}$ :

$$
\begin{aligned}
\Sigma: \dot{x}(t) & =f(x(t), u(t)) \\
y(t) & =h(x(t), u(t))
\end{aligned}
$$

where $y(t) \in \mathbb{R}^{p}$ and $u(t) \in \mathbb{R}^{m}$. By $x\left(t, x_{0}, u\right)$ we will denote the solution at time $t$ of the equation $\dot{x}=f(x, u)$ that satisfies initial condition $x(0)=x_{0}$ and corresponds to control $u$. We will assume that controls are analytic functions of time $t$. By a trajectory of system $\Sigma$ we will mean any triple of functions $y(\cdot),(x(\cdot), u(\cdot))$ defined on some interval that satisfies the equations of $\Sigma$. Then the pair $(x(\cdot), u(\cdot))$ is an inner trajectory and the pair $(y(\cdot), u(\cdot))$ is an external trajectory of $\Sigma_{c}$. The set of all inner (external) trajectories of the system $\Sigma$ forms the inner (external) behavior of the system. It will be denoted by $B^{i}(\Sigma)$ (respectively by $B^{e}(\Sigma)$ ).

By $J(r)$ we will denote the space of all infinite sequences $\left(s^{(i)}\right)_{i \geq 0}, s^{(i)} \in \mathbb{R}^{r}$. We will denote $S=\left(s^{(i)}\right)_{i \geq 0}$. The space $J(r)$ may be interpreted as the space of jets at $t=0$ of smooth functions $s: \mathbb{R} \rightarrow \mathbb{R}^{r}$. Elements $S \in J(s)$ are called infinite jets.

We shall consider real maps defined on the space of infinite jets. We assume that such maps depend only on a finite number of elements of the sequence $S$ (but the number of these elements depends on the given map $\varphi$ ). In this case we say that function $\varphi$ is finitely presented. A map $\phi: J(r) \rightarrow \mathbb{R}^{\tilde{r}}$ is finitely presented if all its components have this property. 
Let us denote by $X \in J(n), Y \in J(p)$ and $U \in J(m)$ - the infinite jets of, respectively, states, outputs and controls. Let us consider two continuous-time systems

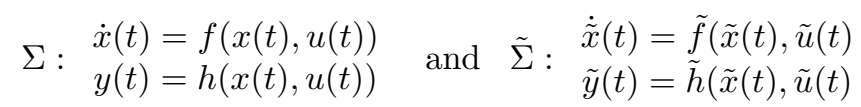

where $x(t) \in \mathbb{R}^{n}, \tilde{x}(t) \in \mathbb{R}^{\tilde{n}}, y(t) \in \mathbb{R}^{p}, \tilde{y}(t) \in \mathbb{R}^{\tilde{p}}, u(t), \tilde{u}(t) \in \mathbb{R}^{m}, t \in$ $\mathbb{R}$. We say that $\Sigma$ and $\tilde{\Sigma}$ are dynamically externally equivalent, if there exist transformations:

$$
\begin{array}{cc}
y=\phi^{e}(\tilde{Y}, \tilde{U}), & u=\psi^{e}(\tilde{Y}, \tilde{U}) \\
\tilde{y}=\tilde{\phi}^{e}(Y, U), & \tilde{u}=\tilde{\psi}^{e}(Y, U)
\end{array}
$$

where $\phi^{e}, \tilde{\phi}^{e}, \psi^{e}, \tilde{\psi}^{e}$ are finitely presented maps of the class $C^{\omega}$ such that the induced maps on pairs $(y(\cdot), u(\cdot))$ and $(\tilde{y}(\cdot), \tilde{u}(\cdot))$ transform external behavior of one system onto external behavior of the second one and are mutually inverse on these behaviors. Then the relation between the external trajectories of both systems can be written as follows:

$$
\begin{array}{r}
y(t)=\phi^{e}\left(\tilde{y}(t), \dot{\tilde{y}}(t), \ldots, \tilde{y}^{(k)}(t), \tilde{u}(t), \dot{\tilde{u}}(t), \ldots, \tilde{u}^{(k)}(t)\right) \\
u(t)=\psi^{e}\left(\tilde{y}(t), \tilde{y}^{\prime}(t), \ldots, \tilde{y}^{(k)}(t), \tilde{u}(t), \tilde{u}^{\prime}(t), \ldots, \tilde{u}^{(k)}(t)\right)
\end{array}
$$

for some $k \geq 0$. Similarly for $(\tilde{y}(t), \tilde{u}(t))$.

We say that the system $\Sigma$ is dynamically externally linearizable if it is dynamically externally equivalent to a linear minimal one (i.e. controllable and observable).

\section{Function universes}

Let $X, Y$ be sets. A partially defined function on $X$ with values in $Y$ is any map $\varphi: A \rightarrow Y$, where $A \subseteq X$ is called domain of $\varphi$ and denoted by $\operatorname{dom} \varphi$. If $\operatorname{dom} \varphi=\mathrm{X}$ then $\varphi$ is global. Let $Y_{X}$ be the set of all partially defined functions on $X$. One can extend any $\varphi \in Y_{X}$ to one defined on $X$ by assigning $\varphi(x)=\emptyset_{0}$ for $x \notin \operatorname{dom} \varphi$. We call $\emptyset_{0}$ the phantom. Now $\operatorname{dom} \varphi=\left\{\mathrm{x} \in \mathrm{X}: \varphi(\mathrm{x}) \neq \emptyset_{0}\right\}$. If $a \in Y, x \in X$ then we set $a_{X}(x):=a$.

Let $A_{n}$ denote the set of functions of class $C^{\omega}$, partially defined on open subsets in $\mathbb{R}^{n}$ with values in $\mathbb{R}$. In particular, $A_{0}$ can be identified with $\mathbb{R} \cup \emptyset_{0}$ and $A_{\mathbb{R}^{n}}=A_{n}$. The topology in $A_{0}$ can be defined as follows: a subset $B \subset A_{0}$ is open if $B=A_{0}$ or $B$ is an open subset in $\mathbb{R}$.

Functions $\varphi, \psi \in Y_{X}$ are matching, if they take on the same values on $\operatorname{dom} \varphi \cap$ $\operatorname{dom} \psi$. Let us consider a set $M \subseteq Y_{X}$ of functions that are matching and define a function $\underline{M} \in Y_{X}: \underline{M}(x)=\emptyset_{0}$ if no function in $M$ is defined at $x$ and $\underline{M}(x)=\varphi(x)$ for any function $\varphi \in M$ defined at $x$. The process of constructing $\underline{M}$ is called amalgamation of the functions of $M$.

Let $\varphi_{1}, \ldots, \varphi_{k} \in \mathbb{R}_{X}$ and $F \in A_{k}$. Then $F \circ\left(\varphi_{1}, \ldots, \varphi_{k}\right)$ is a partially defined function on $X$ given by $\left(F \circ\left(\varphi_{1}, \ldots, \varphi_{k}\right)\right)(x)=F\left(\varphi_{1}(x), \ldots, \varphi_{k}(x)\right)$ for $x \in X$. 
If $\varphi_{i}(x)=\emptyset_{0}$ or $\left(\varphi_{1}(x), \ldots, \varphi_{k}(x)\right) \notin \operatorname{domF}$ then $F\left(\varphi_{0}(x), \ldots, \varphi_{n}(x)\right)=\emptyset_{0}$. The map $\left(\varphi_{1}, \ldots, \varphi_{k}\right) \mapsto F \circ\left(\varphi_{1}, \ldots, \varphi_{k}\right)$ is called a substitution.

A set $\mathcal{U} \subseteq \mathbb{R}_{X}$ containing $0_{X}$ and closed under substitutions and amalgamation is called a function universe on the set $X[\mathrm{Jh}]$. A function subuniverse of the universe $\mathcal{U}$ is a subset $\hat{U} \subset \mathcal{U}$ that is function universe on $X$. If $\mathcal{H} \subset \mathcal{U}$, then function subuniverse generated by $\mathcal{H}$ is the smallest subuniverse of $\mathcal{U}$ containing $\mathcal{H}[1]$.

In a natural way a function universe $\mathcal{U}$ on $X$ induces a topology on $X$ : the open sets have the form $\operatorname{dom} \varphi: \varphi \in \mathcal{U}$.

Let $\mathcal{U}_{1}, \mathcal{U}_{2}$ be the function universes on $X_{1}$ and $X_{2}$ respectively. A map $\tau: \mathcal{U}_{1} \rightarrow \mathcal{U}_{2}$ is a homomorphism of function universes $\mathcal{U}_{1}$ and $\mathcal{U}_{2}$ if

1. $\tau\left(F \circ\left(\varphi_{1}, \ldots, \varphi_{k}\right)\right)=F\left(\tau \varphi_{1}, \ldots, \tau \varphi_{k}\right)$ for $\varphi_{1}, \ldots, \varphi_{k} \in \mathcal{U}_{1}, F \in A_{k}$

2. $\tau(\underline{M})=\tau(M)$ for any matching set $M \subset \mathcal{U}_{1}$

3. $\tau\left(0_{X_{1}}\right)=\overline{0_{X_{2}}}$

If a homomorphism $\tau$ is a bijective map then it is an isomorphism of function universes.

A differential universe is a function universe $\mathcal{U}$ together with an operator $D: \mathcal{U} \rightarrow \mathcal{U}$ that satisfies the chain rule, i.e.

$$
D\left(F\left(\varphi_{1}, \ldots, \varphi_{k}\right)\right)=\sum_{i=1}^{k} \frac{\partial F}{\partial x_{i}}\left(\varphi_{1}, \ldots, \varphi_{k}\right) \cdot D \varphi_{i}
$$

for $F \in A_{k}$ and $\varphi_{1}, \ldots, \varphi_{k} \in \mathcal{U}$. $D$ is called a differential operator. A map $\tau: \mathcal{U}_{1} \rightarrow \mathcal{U}_{2}$ is a homomorphism of differential universes $\left(\mathcal{U}_{1}, D_{1}\right)$ and $\left(\mathcal{U}_{2}, D_{2}\right)$ if it is a homomorphism of universes and $D_{2} \circ \tau=\tau \circ D_{1}$.

Let $\mathcal{U}(n, m)$ denote the function universe of all real finitely presented functions of class $C^{\omega}$ defined on open subsets of $\mathbb{R}^{n} \times J(m)$.

By the differential operator associated with the system $\Sigma$ we will mean the map $D_{\Sigma}: \mathcal{U}(n, m) \rightarrow \mathcal{U}(n, m)$ :

$$
D_{\Sigma}:=\sum_{1 \leq q \leq n} f_{q} \frac{\partial}{\partial x_{q}}+\sum_{i, j} u_{j}^{(i+1)} \frac{\partial}{\partial u_{j}^{(i)}}
$$

where $U=\left\{\left(u_{j}^{(i)}: j=1, \ldots, m\right): i \geq 0\right\} \in J(m)$. The second sum is treated as a formal sum. For $\varphi \in \mathcal{U}(n, m), D_{\Sigma} \varphi$ is a well defined, finitely presented function. If $l \geq 2$, then $D_{\Sigma}^{l} \varphi:=D_{\Sigma}\left(D_{\Sigma}^{l-1} \varphi\right)$. The universe $\mathcal{U}(n, m)$ together with the operator $D_{\Sigma}$ forms a differential universe called the differential universe of the system $\Sigma$ and denoted by $\mathcal{U}_{\Sigma}$. By $\mathcal{H}_{\Sigma}$ we will denote the subunivese of $\mathcal{U}_{\Sigma}$, called differential output universe of the system, generated (as a function universe) by functions $h_{k}, D_{\Sigma}^{i} h_{k}, u_{j}^{(i)} \in \mathcal{U}(n, m), k=1, \ldots, p, j=1, \ldots, m$, $i=0,1, \ldots, u_{j}^{(i)}$ is the coordinate function assigning to $(x, U)$ the $i$-th coordinate of the sequence $U$.

The operator $D_{\Sigma}$ may be interpreted as an abstract vector field on the space 
$\mathbb{R}^{n} \times J(m)$ that corresponds to the following infinite system

$$
\begin{gathered}
\dot{x}=f\left(x, u_{0}\right) \\
\dot{u}_{0}=u_{1} \\
\dot{u}_{1}=u_{2} \\
\cdots \\
y=\stackrel{h}{h}\left(x, u_{0}\right)
\end{gathered}
$$

\section{Indistinguisbability relation}

We say the states $x, z$ are indistinguishable, if for every control $u$

$$
h\left(x_{c}(t, x, u)\right)=h\left(x_{c}(t, z, u)\right)
$$

for every $t \geq 0$ for which both sides are defined. Indistinguishability relation is an equivalence relation. It will be denoted by $\sim$ and the equivalence class of element $x$ by $[x]$.

Let us consider a map $\Pi: \mathbb{R}^{n} \rightarrow \mathbb{R}^{n} / \sim=\mathcal{M}, \Pi(x):=[x] . \mathcal{M}$ is a topological Hausdorff space with quotient topology. On this space let us define the quotient system:

$$
\begin{aligned}
\bar{\Sigma}: \dot{\bar{x}}(t) & =\bar{f}(\bar{x}(t), \bar{u}(t)) \\
\bar{y}(t) & =\bar{h}(\bar{x}(t), \bar{u}(t))
\end{aligned}
$$

where $\bar{x}:=\Pi(x), \bar{u}:=u, \bar{h}(\bar{x}, \bar{u}):=h(x, u) . \bar{f}_{\bar{u}}$ is a vector field on $\mathcal{M}$ defined as a derivation operator by $\bar{f}_{\bar{u}}(\bar{\varphi})(\bar{x}):=f_{u}(\bar{\varphi} \circ \Pi)(x), \bar{\varphi}: \mathcal{M} \rightarrow \mathbb{R}$ and $x \in \Pi^{-1}(\bar{x})$, $f_{u}=f(\cdot, u)$ is identified with a derivation. Then $\left(D_{\bar{\Sigma}} \bar{f}\right)=D_{\Sigma}\left(\bar{\varphi} \circ\left(\Pi, \mathrm{id}_{U}\right)\right)(x, U)$ is the differential operator for the quotient system. Functions $\bar{f}, \bar{h}$ do not depend on the choice of representatives (see [1] for more details).

Remark 4.1. The external behaviors of the original and quotient systems coincide. The original and quotient systems are externally dynamically equivalent. It is enough to put $\bar{y}:=y$ and $\bar{u}:=u$.

Proposition 4.2. $x \sim z \Longleftrightarrow\left(D_{\Sigma}^{k}\right)(x, U)=\left(D_{\Sigma}^{k}\right)(z, U)$ for any $k=, 1,2, \ldots$

Let $\mathcal{H}_{\bar{\Sigma}}$ be the differential output universe of the quotient system. It is generated by functions $D_{\bar{\Sigma}}^{l} \bar{h}_{k}, D_{\bar{\Sigma}}^{l} u_{j}^{(i)} \in \mathcal{U}(\mathcal{M}, J(m)), i, l \in \mathbb{N} \cup\{0\}, k=1, \ldots, p$, $j=1, \ldots, m\}\}$.

Proposition 4.3. The differential output universes $\mathcal{H}_{\Sigma}$ and $\mathcal{H}_{\bar{\Sigma}}$ are isomorphic.

Proof. Let $\Pi_{e}: \mathbb{R}^{n} \times J(m) \rightarrow \mathcal{M} \times J(m), \Pi_{e}(x, U):=(\Pi(x), U)$. Let $\tau: \mathcal{H}_{\Sigma} \rightarrow \mathcal{H}_{\bar{\Sigma}}$, $(\tau \varphi)(x, U):=\left(\varphi \circ \Pi_{e}\right)(x, U)$. Then $\tau=\left.\Pi_{e}^{*}\right|_{\mathcal{H}_{\bar{\Sigma}}}$ and from Proposition $\left.4.2 \Pi_{e}^{*}\right|_{\mathcal{H}_{\bar{\Sigma}}}$ is one-to-one.

Let us consider a homomorphism of function universes

$$
\Pi_{e}^{*}: \mathbb{R}_{\mathcal{M} \times J(m)} \rightarrow \mathbb{R}_{\mathbb{R}^{n} \times J(m)} .
$$


Then for any $\bar{\varphi} \in \mathcal{H}_{\bar{\Sigma}}$

$$
\begin{aligned}
\Pi_{e}^{*}\left(D_{\bar{\Sigma}} \bar{h}\right)(x, U)=\left(D_{\bar{\Sigma}} \bar{h}\right)(\Pi(x), U) & =D_{\Sigma}\left(\bar{h} \circ\left(\Pi, \operatorname{id}_{U}\right)\right)(x, U)= \\
\left.\frac{d}{d t}\right|_{t=0}(h(x(t, x, u))) & =\left(D_{\Sigma} h\right)(x, U) .
\end{aligned}
$$

Similarly we can show that $\Pi_{e}^{*}\left(D_{\Sigma}^{l} \bar{h}\right)(x, U)=\left(D_{\Sigma}^{l} h\right)(x, U)$ for $l=1,2, \ldots$ Moreover,

$$
\begin{gathered}
\Pi_{e}^{*}\left(D_{\bar{\Sigma}} \bar{u}_{j}^{(i)}\right)(x, U)=\left(D_{\bar{\Sigma}} \bar{u}_{j}^{(i)}\right)(\Pi(x), U)= \\
D_{\Sigma}\left(u_{j}^{(i)} \circ\left(\Pi, \operatorname{id}_{U}\right)\right)(x, U)=\left(D_{\Sigma} u_{j}^{(i)}\right)(x, U)
\end{gathered}
$$

and similarly, $\Pi_{e}^{*}\left(D_{\bar{\Sigma}}^{l} \bar{u}_{j}^{(i)}\right)(x, U)=\left(D_{\Sigma}^{l} u_{j}^{(i)}\right)(x, U)$ for $l=1,2, \ldots$ Because functions $\bar{h}, D_{\bar{\Sigma}}^{l} \bar{h}, \bar{u}_{j}^{(i)}$ are generators of $\mathcal{H}_{\bar{\Sigma}}$ and $h, D_{\Sigma}^{l} h, u_{j}^{(i)}$ are generators of $\mathcal{H}_{\Sigma}$, hence $\Pi_{e}^{*}\left(\mathcal{H}_{\bar{\Sigma}}\right)=\mathcal{H}_{\Sigma}$. Bijectivity of the map $\Pi_{e}^{*}$ implies that $\tau=\left.\Pi_{e}^{*}\right|_{\mathcal{H}_{\bar{\Sigma}}}$ is an isomorphism of function universes. Moreover, because

$$
\begin{aligned}
& \left(D_{\Sigma}(\tau \varphi)(x, U)=\frac{d}{d t} \mid t=0\right. \\
& =\left(D_{\bar{\Sigma}} \varphi\right)\left(\Pi_{e}(x, U)\right)=\tau\left(D_{\Sigma} \varphi\right)(x, U)=\left.\frac{d}{d t}\right|_{t=0} \varphi\left(\Pi_{e}(x, U)\right) \\
& \Pi_{e}^{*}\left(D_{\Sigma} \varphi\right)(x, U)
\end{aligned}
$$

hence $\tau$ is an isomorphism of the difference universes.

\section{Conditions of equivalence}

The control system is observable if any two distinct points are indistinguishable. It can be noticed that the quotient system is observable.

Let us consider a map $T: \mathcal{M} \times J(m) \rightarrow J(p, m)$, where $J(p, m)$ denotes the space of jets of outputs and controls, defined as follows:

$$
T(\bar{x}, \bar{U})=\left(\bar{h}, D_{\bar{\Sigma}} \bar{h}, D_{\bar{\Sigma}}^{2} \bar{h}, \ldots, \bar{u}, D_{\bar{\Sigma}} \bar{u}, D_{\bar{\Sigma}}^{2} \bar{u}, \ldots\right)(\bar{x}, \bar{U})
$$

where $\bar{U}=U \in J(m)$. Let

$$
\left.T\right|_{k}(\bar{x}, \bar{U})=\left(\bar{h}, D_{\bar{\Sigma}} \bar{h}, \ldots, D_{\bar{\Sigma}}^{k-1} \bar{h}, \bar{u}, D_{\bar{\Sigma}} \bar{u}, \ldots, D_{\bar{\Sigma}}^{k-1} \bar{u}\right)(\bar{x}, \bar{U}) .
$$

For fixed $\bar{U}$

$$
T_{\bar{U}}(\bar{x})=\left(\bar{h}, D_{\bar{\Sigma}} \bar{h}, D_{\bar{\Sigma}}^{2} \bar{h}, \ldots\right)(\bar{x}, \bar{U})
$$

and

$$
T_{\left.\bar{U}\right|_{k}}(\bar{x})=\left(\bar{h}, D_{\bar{\Sigma}} \bar{h}, \ldots, D_{\bar{\Sigma}}^{k-1} \bar{h}\right)(\bar{x}, \bar{U}) .
$$

We say that $T_{U}$ is an immersion at point $\bar{x}$ if there exists $k \in \mathbb{N}$ such that $T_{\left.\bar{U}\right|_{k}}$ is an immersion at $\bar{x}$.

From now on we shall assume the following

A1. $\mathcal{M}$ ia a manifold of the class $C^{\omega}$.

A2. For every $\bar{x} \in \mathcal{M}$ and every $\bar{U} \in J(m)$ there exists $k \in \mathbb{N}$ such that $\left.T\right|_{k}$ is a proper map on some neighborhood $(\bar{x}, \bar{U})$.

A3. For every $\bar{U} \in J(m)$ the map $\left.T\right|_{\bar{U}}$ is an imbedding. 
Theorem 5.1. Under conditions A1-A3 continuous-time systems $\Sigma$ and $\tilde{\Sigma}$ are externally dynamically equivalent if and only if their differential output universes $\mathcal{H}_{\Sigma}$ and $\mathcal{H}_{\Sigma}$ are isomorphic.

\section{Linearization}

Let us recall that linear systems

$$
\Lambda: \begin{aligned}
& \dot{x}=A x+B u \\
& y=C x+D u
\end{aligned} \quad \text { and } \quad \tilde{\Lambda}: \begin{aligned}
& \dot{\tilde{x}}=\tilde{A} \tilde{x}+\tilde{B} \tilde{u} \\
& \tilde{y}=\tilde{C} \tilde{x}+\tilde{D} \tilde{u}
\end{aligned}
$$

or shortly: $(A, B, C, D),(\tilde{A}, \tilde{B}, \tilde{C}, \tilde{D})$ are equivalent with respect to a linear static state feedback if there exist matrices $T, F, G, T, G$ - invertible, such that the inner trajectories $(\tilde{X}, \tilde{U})$ of $\tilde{\Lambda}$ are transformed onto inner trajectories of $\Lambda$ by transformations

$$
x=T \tilde{x}, \quad u=F \tilde{x}+G \tilde{u},
$$

and vice versa.

Lemma 6.1. If two observable linear systems $(A, B, C, D)$ and $(\tilde{A}, \tilde{B}, \tilde{C}, \tilde{D})$ are equivalent with respect to a linear static state feedback transformations then they are equivalent with respect to a linear external dynamic transformations.

Proof. It is clear that for an observable linear system the state $x(t)$ may be expressed as a function of $y^{(k)}(t)$ and $u^{(k)}(t)$, where $k=0,1, \ldots, n-1$. Thus $x=\gamma(Y, U)$ with $\gamma$ a finitely presented function, and similarly $\tilde{x}=\tilde{\gamma}(\tilde{Y}, \tilde{U})$.

The static state transformations

$$
x=T \tilde{x}, \quad u=F \tilde{x}+G \tilde{u}
$$

$F, G$ - invertible, give the corresponding external dynamic transformations:

$$
y=C T \tilde{\gamma}(\tilde{Y}, \tilde{U})+D F \tilde{\gamma}(\tilde{Y}, \tilde{U}) \text { and } u=F \tilde{\gamma}(\tilde{Y}, \tilde{U}) .
$$

Similarly in the other way.

We say that a differential universe $(\mathcal{U}, D)$ is free if there exist functions $\omega_{1}, \ldots, \omega_{r} \in \mathcal{U}$ such that

1. for any finitely presented function $\nu: J(p) \rightarrow A_{0}$ of class $C^{\omega}: \nu \circ W \equiv 0 \Rightarrow$ $\nu \equiv 0$, where $W=\left\{D^{j} w_{i}\right\}_{i=1, \ldots, r ; j=0,1, \ldots}$

2. for any $\varphi \in \mathcal{U}$ there exists a finitely presented function $\nu: J(p) \rightarrow A_{0}$ of class $C^{\omega}$ such that $\varphi=\nu \circ W$.

The first condition means that there is no functional relation between $\omega_{i}$, $i=1, \ldots, r$ and their differentials. The second one says that any function from universe $\mathcal{U}$ can be expressed as a function on $\omega_{i}$ 's and their differentials. Functions $\omega_{1}, \ldots, \omega_{r}$ are called free generators of universe $(\mathcal{U}, D)$.

Lemma 6.2. Let $\Lambda$ be a controllable linear system. The differential output universe $\mathcal{H}_{\Lambda}$ of this system is free. 
The proof is similar as in discrete-time case [8].

Theorem 6.3. The system $\Sigma$ is externally dynamically linearizable if and only if $\mathcal{H}_{\Sigma}$ is a free universe.

Proof. Implication " $\Rightarrow "$ follows from Theorem 5.1 and Lemma 6.2. To prove the converse let us consider the systems

$$
\Sigma: \begin{aligned}
& \dot{x}=f(x, u) \\
& y=h(x, u)
\end{aligned} \quad \text { and } \quad \Lambda: \begin{aligned}
& \dot{\tilde{x}}_{i}=\tilde{u}_{i} \\
& \tilde{y}_{i}=\tilde{x}_{i}
\end{aligned}
$$

where $i=1, \ldots, m, u \in \mathbb{R}^{m}$. Let $\omega_{1}, \ldots, \omega_{m}$ be free generators of the universe $\mathcal{H}_{\Sigma}$. Let us consider a map $\tau: \mathcal{H}_{\Sigma} \rightarrow \mathcal{H}_{\Lambda}$ defined by $\tau\left(w_{i}\right)=\tilde{x}_{i}, \tau\left(D_{\Sigma} w_{i}\right)=\tilde{u}_{i}$. The second condition of free universe allows us to extend $\tau$ on the whole universe $\mathcal{H}_{\Sigma}$ via substitutions. It is clear that $\tau$ is "onto". If $\alpha$ is a finitely presented function, then

$$
\begin{gathered}
\tau\left(\alpha\left(w_{1}, \ldots, w_{m}, D_{\Sigma} w_{1}, \ldots, D_{\Sigma} w_{m}, D_{\Sigma}^{2} w_{1}, \ldots, D_{\Sigma}^{2} w_{m}, \ldots\right)\right)= \\
\alpha\left(\tau w_{1}, \ldots, \tau w_{m}, \tau\left(D_{\Sigma} w_{1}\right), \ldots, \tau\left(D_{\Sigma} w_{m}\right), \tau\left(D_{\Sigma}^{2} w_{1}\right), \ldots, \tau\left(D_{\Sigma}^{2} w_{m}, \ldots\right)\right)= \\
\alpha\left(\tilde{x}_{1}, \ldots, \tilde{x}_{m}, \tilde{u}_{1}^{0}, \ldots, \tilde{u}_{m}^{0}, \tilde{u}_{1}^{1}, \ldots, \tilde{u}_{m}^{1}, \ldots\right) \equiv 0 \Rightarrow \alpha \equiv 0 .
\end{gathered}
$$

Hence $\tau$ is "1-1". Moreover because $\tau\left(D_{\Sigma} w_{i}\right)=\tilde{u}_{i}=D_{\Lambda} \tilde{x}_{i}=D_{\Lambda}\left(\tau w_{i}\right)$ then $\tau$ commutes with differential operators. Hence $\tau: \mathcal{H}_{\Sigma} \rightarrow \mathcal{H}_{\Lambda}$ is an isomorphism and systems $\Sigma$ and $\Lambda$ are externally dynamically equivalent.

Example 6.4. Let us consider the system $\dot{x}=u, y=\sin x$. The differential output universe contains functions $\sin x, u \cos x$ and $u$. This allows to produce $x$ via substitutions and amalgamations. More precisely, for $x \in(-\pi / 2+2 k \pi, \pi / 2+$ $2 k \pi), x=\arcsin (\sin x)+2 k \pi$, and for $x \in(2 k \pi,(2 k+1) \pi), x=\arccos (\cos x)+$ $2 k \pi$. The set of all these functions is matching and amalgamates to $x$ on the entire $\mathbb{R}$. This is a free generator of the differential output universe, so the system is externally dynamically linearizable. The above calculations show in fact how to define the the external dynamic transformations that linearize the system.

Remark 6.5. The above example, though very simple, shows the essential features of the "universal" approach. We use substitutions and amalgamations which cannot be used in the language of (differential) algebras or fields. The need for substitutions may be seen in Jakubczyk's original definition of free generators of the differential algebra of a system without output. The definition is essentially the same as here, so it has some "universal" features.

\section{References}

[1] Z. Bartosiewicz, J. Johnson, Systems on universe spaces, Acta Applicandae Mathematicae, 34 (1994). 
[2] Z. Bartosiewicz, E. Pawłuszewicz, External equivalence of unobservable discrete-time systems, Proceedings of International Control Conference NOCOLS'98, Eschencde, Netherlands, 1998.

[3] B. Charlet, J. Levine and R. Marino, Sufficient conditions for dynamic state feedback linearization, SIAM J. Control and Optimization 29 (1991), 38-57.

[4] B. Jakubczyk, Dynamic feedback equivalence of nonlinear control systems, preprint

[5] J. Johnson, A generalized global differential calculus I, Cahiers Top. et Geom. Diff. XXVII(1986)

[6] Ph. Martin, R. M. Murray, P. Rouchon, Flat systems, Plenary Lectures and Mini-Courses, European Control Conference ECC'97, G. Bastin and M. Gevers, Eds., Brussels, 1997

[7] E. Pawłuszewicz, Z. Bartosiewicz, External Dynamic Feedback Equivalence of Observable Discrete-time Control Systems, Proc. of Symposia in Pure Mathematics, vol.64, American Mathematical Society, Providence, Rhode Island, 1999

[8] E. Pawłuszewicz, Dynamic linearization of input-output discrete-time systems, in: Proceedings of the International Conference UKACC Control'96, Exeter, UK, 1996.

[9] J.-B. Pomet, A differential geometric setting for dynamic equivalence and dynamic linearization, in: Geometry in Nonlinear Control and Differential Inclusions, Banach Center Publications, vol.32, Institute of Mathematics, Polish Academy of Sciences, Warsaw, Poland 1995 